\title{
MIMO Signal Detection Using Neyman Pearson Signal Detection
}

\author{
Deepali Kamble ${ }^{1}$, Prof.C.A.Manjare ${ }^{2}$ \\ Department of E\&TC Engineering, JSPMs, Jayawantrao Sawant College of Engg, Hadapsar, Pune, India ${ }^{1,2}$
}

\begin{abstract}
In current scenario, the demand for wireless communication is increasing drastically. A wireless system has number of advantages over its wired counterpart including allowing a communication link to be set up quickly without the difficulty and expense of installing data transmission lines. The wireless communications industry has experienced an explosive growth in the last decade. One of the most promising spectrums an efficient technique is multiple-inputmultiple-output (MIMO) systems that employ multiple transmits and receives antennas. The multiple inputs multiple outputs (MIMO) radar system transmits $\mathrm{M}$ antennas and receives $\mathrm{N}$ antennas. In this proposed system first step can be initially derive the diversity gain for a signal present versus signal absent scalar hypothesis test statistic and for a vector signal present versus vector signal absent hypothesis test. The MIMO radar system, used to detect a target composed of $\mathrm{Q}$ random scatterers with possibly non-Gaussian reflection coefficients in the presence of possibly non-Gaussian clutter-plus-noise. Diversity gain for the MIMO radar system is dependent on the cumulative distribution function (CDF). In this maximum possible diversity gain can be achieved for non orthogonal waveforms.
\end{abstract}

Keywords: Neyman-Pearson detection, Diversity gain, multiple-input multiple output (MIMO) system, signal space.

\section{INTRODUCTION}

Multiple-input multiple-output (MIMO) radar is an emerging topic that draws upon research in the fields of communications and radar. In this system several transmitting antennas and one receiving antenna is present [4]-[6]. This is also known as transmitting diversity. The transmit diversity is far more advantageous in comparison to the receive diversity. This is due to the fact that in general the number of receivers is greater than the number of transmitters. The transmit diversity is a modern phenomenon. In this case, the same data is transmitted redundantly over two antennas. This method has the advantage that the multiple antennas and redundancy coding is moved from the mobile user end to the base station, where these technologies are simpler and cheaper to implement. MIMO radars that employ dispersed antennas to transmit various waveforms can obtain a larger diversity gain than the conventional phased array radar.

When the Neyman-Pearson criterion is employed, we fix the false alarm probability. The diversity gain is defined as the negative of the slope of the miss probability versus signal-to-clutter-plus-noise ratio (SCNR) for the high SCNR region when a logarithmic scale is employed for both axes[5]. Assuming linear decay of the miss probability for sufficiently large SCNR when such scales are employed, large diversity gain implies good target detection performance for sufficiently high SCNR and fixed probability of false alarm. Intuitively, diversity gain tells us about the value of the information we get from multiple looks (from several antennas, frequencies, or retransmissions, etc.). In particular, the calculation of diversity gain typically shows that multiple looks tend to increase the diversity gain. This seems reasonable for large enough SCNR, where each individual look should help in making a correct decision[2]. Of course, no scalar performance measure can completely describe everything about performance as we change multiple parameters
(SCNR, probability of false alarm). However, diversity gain is very useful for cases with high SCNR. In this paper, we consider non-orthogonal waveforms for results. At first, we consider a signal-present versus signal-absent scalar hypothesis test statistic under the test statistic contains only. Next, we formulate a vector hypothesis testing problem where we attempt to distinguish between clutter-plus-noise only, and a linearly transformed version of a possibly non-Gaussian signal vector plus this clutterplus-noise. We consider a class of test statistics, including the optimum test for Gaussian signal and Gaussian clutterplus-noise. For the vector hypothesis testing problem, the signal part of the resulting test statistic, formally called $u$ is decomposed into $\mathrm{L}$ terms. Then, we apply these results to study the diversity gain for a MIMO radar system with $\mathrm{M}$ transmit antennas and $\mathrm{N}$ receive antennas which is used to detect a target composed of $\mathrm{Q}$ scatterers. It is shown that the maximum diversity gain can be achieved under certain conditions. These results generalize the Gaussian clutter-plus-noise and reflection coefficients.

The rest of the paper is organized as follows. Section II derives the concept of Multiple Input Multiple Outputs (MIMO) and diversity gain. Section III investigates the Neyman-Pearson Detectors method. Numerical results are presented in Section IV. Conclusions are drawn in Section $\mathrm{V}$.

\section{Multiple InPut Multiple OUtPuts (MIMO)}

A channel may be affected by fading and this will impact the signal to noise ratio. In turn this will impact the error rate, assuming digital data is being transmitted. The principle of diversity is to provide the receiver with multiple versions of the same signal. If these can be made to be affected in different ways by the signal path, the probability that they will all be affected at the same time is considerably reduced. Accordingly, diversity helps to 
stabilize a link and improves performance, reducing error rate.

Several different diversity modes are available and provide a number of advantages:

Time diversity: Using time diversity, a message may be transmitted at different times, e.g. using different timeslots and channel coding.

Frequency diversity: This form of diversity uses different frequencies. It may be in the form of using different channels, or technologies such as spread spectrum / OFDM.

Space diversity: Space diversity used in the broadest sense of the definition is used as the basis for MIMO. It uses antennas located in different positions to take advantage of the different radio paths that exist in a typical terrestrial environment.

MIMO is effectively a radio antenna technology as it uses multiple antennas at the transmitter and receiver to enable a variety of signal paths to carry the data, choosing separate paths for each antenna to enable multiple signal paths to be used.

One of the core ideas behind MIMO wireless systems space-time signal processing in which time (the natural dimension of digital communication data) is complemented with the spatial dimension inherent in the use of multiple spatially distributed antennas, i.e. the use of multiple antennas located at different points. Accordingly MIMO wireless systems can be viewed as a logical extension to the smart antennas that have been used for many years to improve wireless.

It is found between a transmitter and a receiver, the signal can take many paths. Additionally by moving the antennas even a small distance the paths used will change. The variety of paths available occurs as a result of the number of objects that appear to the side or even in the direct path between the transmitter and receiver. Previously these multiple paths only served to introduce interference. By using MIMO, these additional paths can be used to advantage. They can be used to provide additional robustness to the radio link by improving the signal to noise ratio, or by increasing the link data capacity.

The two main formats for MIMO are given below:

\section{Spatial diversity:}

Spatial diversity used in this narrower sense often refers to transmit and receive diversity. These two methodologies are used to provide improvements in the signal to noise ratio and they are characterized by improving the reliability of the system with respect to the various forms of fading.

\section{Spatial multiplexing:}

This form of MIMO is used to provide additional data capacity by utilizing the different paths to carry additional traffic, i.e. increasing the data throughput capability.

As a result of the use multiple antennas, MIMO wireless technology is able to considerably increase the capacity of a given channel while still obeying Shannon's law. By increasing the number of receive and transmit antennas it is possible to linearly increase the throughput of the channel with every pair of antennas added to the system. This makes MIMO wireless technology one of the most important wireless techniques to be employed in recent years. As spectral bandwidth is becoming an ever more valuable commodity for radio communications systems, techniques are needed to use the available bandwidth more effectively. MIMO wireless technology is one of these techniques.

There are a number of different MIMO configurations or formats that can be used. These are termed SISO, SIMO, MISO and MIMO. These different MIMO formats offer different advantages and disadvantages - these can be balanced to provide the optimum solution for any given application.

The different MIMO formats - SISO, SIMO, MISO and MIMO require different numbers of antennas as well as having different levels of complexity. Also dependent upon the format, processing may be needed at one end of the link or the other - this can have an impact on any decisions made.

Wireless communication industry has recently turned to a strategy called Multiple-Input Multiple-Output (MIMO). MIMO is the single most important wireless technology as of today. MIMO is a technology evolution where both ends of the wireless link are equipped with antenna array as shown in fig. 1.

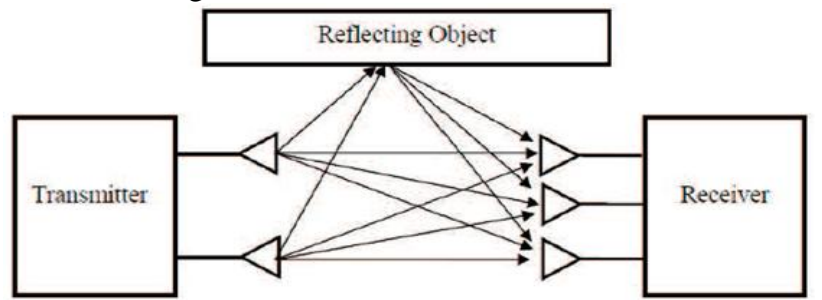

Fig. 1. Block diagram of MIMO system

is designated to the use of multiple antennas in both the transmitter and receiver stations. The new MIMO concept has been originally proposed to improve the communication performance in wireless systems. The Multiple Input Multiple Output Systems is as shown in Fig.2.

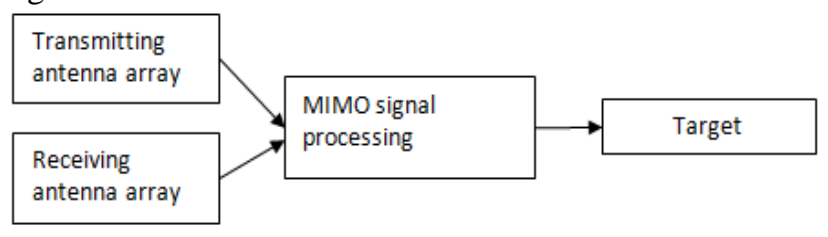

Fig.2. Multiple Input Multiple Output Systems

The performance of a communication system can be heavily disrupted by the multipath effects and doppler spreading effect. Diversity implementations can help to counteract this undesired effect in many ways. These ways can be either built in transmission or reception, and the usage only depends on the increment cost that we can assume or the environment conditions. Diversity 
techniques make port from the random nature of the radio We see that the likelihood ratio statistic was optimal for channel, by sending the information in deferent channels, testing between two simple hypotheses. The test simply which means that we have at the output deferent versions compares the likelihood ratio to a threshold. The "optimal" of the same signal. There are various types of diversity like as Space diversity, Frequency diversity, Time diversity, Polarization diversity and Receiver diversity.

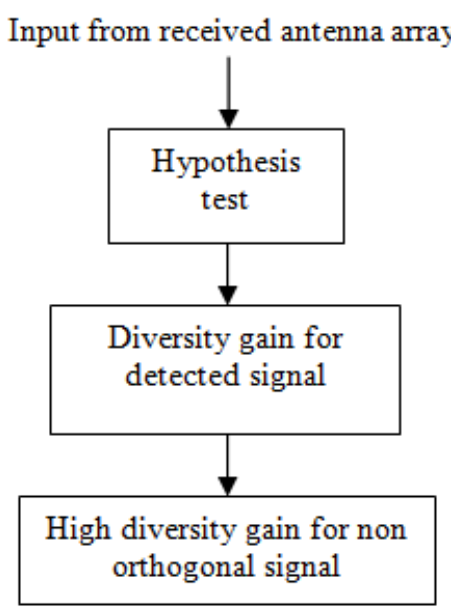

Fig.3. Block diagram of signal processing in MIMO system.

\section{Hypothesis test:}

Input coming from antenna array is given to the hypothesis test. Hypothesis test is done using Neyman Pearson signal detection. First hypothesis test for scalar signal and second hypothesis test for vector signal.

2. Diversity gain for detected signal:

After detecting the signal then calculate the diversity gain. The diversity gain is defined as the negative of the slope of the miss probability versus signal-to-clutter-plus-noise ratio (SCNR) for the high SCNR region when a logarithmic scale is employed for both axes. Diversity gain is main advantages of MIMO system. Diversity gain improves the performance of communication system.

3. High diversity gain for non orthogonal signal

In this main aim of project is to calculate diversity gain for non orthogonal signal. After calculating the diversity gain of non orthogonal signal, it shows that diversity gain for non orthogonal signal is higher than orthogonal signal.

\section{III.NEYMAN-PEARSON DETECTORS METHOD}

Neyman-Pearson's hypothesis makes sense when there are two disjoint alternatives between which we decide, as well as the risk of a Type I error. Explanation: Consider a pvalue is the probability of getting a sample statistic (say, a sample mean). Neyman \& Pearson thought you could use the p-value as part of a formalized decision making process. At the end of your investigation, you have to either reject the null hypothesis, or fail to reject the null hypothesis. In addition, the null hypothesis could be either true or not true. Thus, there are four theoretical possibilities (although in any given situation, there are just two): you could make a correct decision (fail to reject a true--or reject a false--null hypothesis), or you could make a type I or type II error (by rejecting a true null, or failing to reject a false null hypothesis, respectively). threshold is a function of the prior probabilities and the costs assigned to different errors. The choice of costs is subjective and depends on the nature of the problem, but the prior probabilities must be known. Unfortunately, often the prior probabilities are not known precisely, and thus the correct setting for the threshold is unclear.

To deal with this, consider an alternative design specification. Let's design a test that minimizes one type of error subject to a constraint on the other type of error. This constrained optimization criterion does not require knowledge of prior probabilities nor cost assignments. It only requires a specification of the maximum allowable value for one type of error, which is sometimes even more natural than assigning costs to the different errors. A classic result due to Neyman and Pearson shows that the solution to this type of optimization is again a likelihood ratio test.

Assume that we observe a random variable distributed according to one of two distributions.

$$
\begin{aligned}
& H_{0}: X \sim p_{0} \\
& H_{1}: X \sim p_{1}
\end{aligned}
$$

In many problems, $\mathrm{H} 0$ is consider being a sort of baseline or default model and is called the null hypothesis. H1 is a different model and is called the alternative hypothesis. If a test chooses $\mathrm{H} 1$ when in fact the data were generated by $\mathrm{HO}$ the error is called a false-positive or false-alarm, since we mistakenly accepted the alternative hypothesis. The errors of deciding $\mathrm{H} 0$ when $\mathrm{H} 1$ was the correct model is called a false-negative or miss.

Let $\mathrm{T}$ denote a testing procedure based on an observation of $\mathrm{X}$, and let RT denote the subset of the range of $\mathrm{X}$ where the test chooses H1. The probability of a false-positive is denoted by

$$
P_{0}\left(R_{T}\right):=\int_{R_{T}} p_{0}(x) d x
$$

The probability of a false-negative is $1-\mathrm{P} 1(\mathrm{RT})$, where

$$
P_{1}\left(R_{T}\right):=\int_{R_{T}} p_{1}(x) d x
$$

is the probability of correctly deciding $\mathrm{H} 1$, often called the probability of detection.Consider likelihood ratio tests of the form

$$
\frac{p_{1}(x)}{p_{o}(x)} \underset{H_{0}}{\stackrel{H_{1}}{\gtrless}} \lambda .
$$

The subset of the range of $\mathrm{X}$ where this test decides $\mathrm{H} 1$ is denoted

$$
R_{L R}(\lambda):=\left\{x: p_{1}(x)>\lambda p_{0}(x)\right\},
$$

and therefore the probability of a false-positive decision is 


$$
\begin{aligned}
& P_{0}\left(R_{L R}(\lambda)\right):=\int_{R_{L R}(\lambda)} p_{0}(x) d x \\
& =\int_{\left\{x: p_{1}(x)>\lambda p_{0}(x)\right\}} p_{0}(x) d x
\end{aligned}
$$

This probability is a function of the threshold $\lambda$ the set RLR $(\lambda)$ shrinks/grows as $\lambda$ increases/decreases. We can select $\lambda$ to achieve a desired probability of error.

Lemma 1 (Neyman-Pearson)

Consider the likelihood ratio test

$$
\frac{p_{1}(x)}{p_{o}(x)} \underset{H_{0}}{\gtrless} \lambda
$$

With $\lambda>0$ chosen so that P0 $(\operatorname{RLR}(\lambda))=\alpha$. There does not exist another test T with P0 (RT) $\leq \alpha$ and P1 (RT) $>$ P1 $(\operatorname{RLR}(\lambda))$. That is, the LRT is the most powerful test with probability of false-positive less than or equal to $\alpha$. Proof. Let T be any test with P0 (RT) $=\alpha$ and let NP denote the LRT with $\lambda$ chosen so that P0 $(\operatorname{RLR}(\lambda))=\alpha$. To simplify the notation we will denote use RNP to denote the region RLR ( $\lambda$ ). For any subset $R$ of the range of $X$ define

$$
P_{i}(R):=\int_{R} p_{i}(x) d x,
$$

This is simply the probability of $\mathrm{X} \varepsilon \mathrm{R}$ under hypothesis Hi. Note that

$$
\begin{aligned}
P_{i}\left(R_{N P}\right) & =P_{i}\left(R_{N P} \cap R_{T}\right)+P_{i}\left(R_{N P} \cap R_{T}^{c}\right) \\
P_{i}\left(R_{T}\right) & =P_{i}\left(R_{N P} \cap R_{T}\right)+P_{i}\left(R_{N P}^{c} \cap R_{T}\right)
\end{aligned}
$$

Where the superscript $\mathrm{c}$ indicates the complement of the set. By assumption $\mathrm{P} 0(\mathrm{RNP})=\mathrm{P} 0(\mathrm{RT})=\alpha$, therefore

$$
P_{0}\left(R_{N P} \cap R_{T}^{c}\right)=P_{0}\left(R_{N P}^{c} \cap R_{T}\right)
$$

Now, we want to show

$$
P_{1}\left(R_{N P}\right) \geq P_{1}\left(R_{T}\right)
$$

This holds if

$$
P_{1}\left(R_{N P} \cap R_{T}^{c}\right) \geq P_{1}\left(R_{N P}^{c} \cap R_{T}\right) .
$$

To see that this is indeed the case,

$$
\begin{aligned}
P_{1}\left(R_{N P} \cap R_{T}^{c}\right) & =\int_{R_{N P} \cap R_{T}^{c}} p_{1}(x) d x \\
& \geq \lambda \int_{R_{N P} \cap R_{T}^{c}} p_{o}(x) d x \\
& =\lambda P_{o}\left(R_{N P} \cap R_{T}^{c}\right) \\
& =\lambda P_{o}\left(R_{N P}^{c} \cap R_{T}\right) \\
& =\lambda \int_{R_{N P}^{c} \cap R_{T}} p_{o}(x) d x \\
& \geq \int_{R_{N P}^{c} \cap R_{T}} p_{1}(x) d x \\
& =P_{1}\left(R_{N P}^{c} \cap R_{T}\right) .
\end{aligned}
$$

The probability of a false-positive is also called the probability of false-alarm, which we will denote by PFA in the following examples. We will also denote the probability of detection (1- probability of a false-negative) by PD. The NP test maximizes PD subject to a constraint on PFA.

\section{IV.NUMERICAL RESULTS}

Consider the scenario shown in Fig. 4. The radar system has $\mathrm{M}=2$ transmit antennas located at $(\mathrm{x} 1 \mathrm{t}, \mathrm{y} 1 \mathrm{t})=(2,-2) \mathrm{km}$ and $(\mathrm{x} 2 \mathrm{t}, \mathrm{y} 2 \mathrm{t})=(6,-4) \mathrm{km}$ and $\mathrm{N}=2$ receive antennas located at $(\mathrm{x} 1 \mathrm{r}, \mathrm{y} 1 \mathrm{r})=(8,2) \mathrm{km}$ and $(\mathrm{x} 2 \mathrm{r}, \mathrm{y} 2 \mathrm{r})=(4,0) \mathrm{km}$. The waveforms emitted from these two transmitters are $\mathrm{s} 1(\mathrm{t})$ and $\mathrm{s} 2(\mathrm{t})$ respectively. The $\mathrm{Q}$ scatterers constituting the target are uniformly distributed over $[0.3,1] \mathrm{X}$ $[9.4,10.5] \mathrm{km} 2$. Now we present a few numerical examples based on the received signal model, where the Gaussian optimum detector is employed.

In each example, the probability of miss versus SCNR curve is obtained from 100000 Monte Carlo simulations per SCNR, and the resulting diversity gains are compared with those calculated using the corresponding theorem from the previous sections. Throughout this section we assume each scatterer has a statistically independent scattering coefficient.

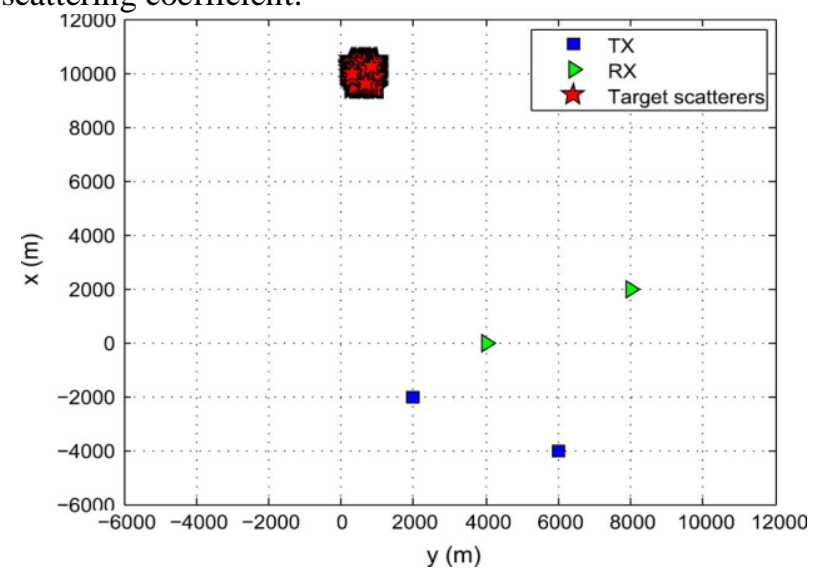

Fig. 4. System setup.

Gaussian Reflections and Non-Gaussian Clutter-Plus-

Noise: The experiments in Fig. 5 are repeated for nonGaussian clutter-plus-noise, while the other parameters remain the same. Assuming the clutter-plus-noise components are statistically independent with lognormal10 distribution with parameters the miss probability versus SCNR curves for the three cases are plotted in Fig. 3. The solid curve with points marked with diamonds shows the case for orthogonal (in the sense of [14]) noisefree received waveforms at each receiver (due to target reflection), and $\mathrm{Q}=1500$ where the diversity gain $\mathrm{D}=4$ read from the figure agrees with the $\mathrm{d}=\mathrm{NM}=4$ using Theorem The dotted curve with points marked with circles represents the case for orthogonal (in the sense of [14]) noise-free received waveforms at each receiver (due to target reflection), and $\mathrm{Q}=2$ where the diversity gain $\mathrm{d}=2$ observed is consistent with the computed result $\mathrm{d}=\mathrm{Q}=2$ from Theorem 2 . 


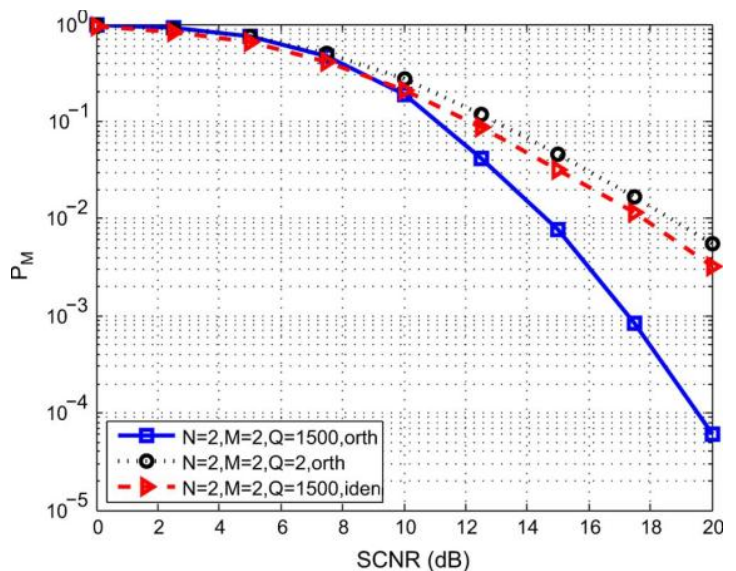

Fig. 5. Probability of miss versus SCNR for three difference cases under the condition of Gaussian reflections and Gaussian clutter-plus-noise.

Fig. 6 again validates Theorem 2, and it also shows that the diversity gain of the considered test does not change even if the actual clutter-plus-noise confronted is nonGaussian. As expected, it is seen that the miss probabilities shown in Fig. 6 are uniformly larger than the corresponding results in Fig. 5, since the test is optimum for Gaussian clutter-plus-noise but not for the nonGaussian clutter-plus-noise.

Spatial diversity used in this narrower sense often refers to transmit and receive diversity. These two methodologies are used to provide improvements in the signal to noise ratio and they are characterized by improving the reliability of the system with respect to the various forms of fading.

Input coming from antenna array is given to the hypothesis test. Hypothesis test is done using Neyman Pearson signal detection. First hypothesis test for scalar signal and second hypothesis test for vector signal. The choice of costs is subjective and depends on the nature of the problem, but the prior probabilities must be known. Unfortunately, often the prior probabilities are not known precisely, and thus the correct setting for the threshold is unclear.

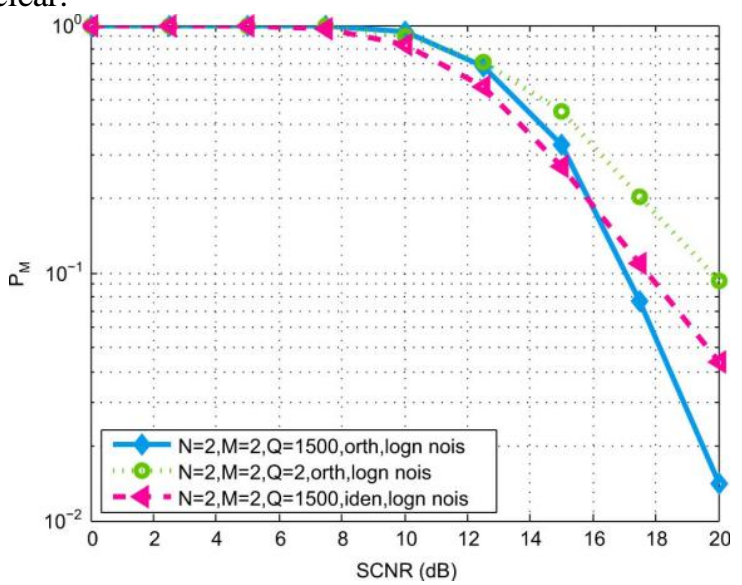

Fig. 6. Probability of miss versus SCNR for three difference cases under the condition of Gaussian reflections and log-normal clutter-plus-noise. Non-Gaussian Reflections and Non-Gaussian ClutterPlus-Noise: Again, the experiments in Fig. 5 are repeated. This time, both non-Gaussian reflections and nonGaussian clutter-plus-noise are considered. While all the other parameters remain the same as those employed in
Fig. 5, we assume the noise-free received waveforms at each receiver (due to target reflection) are orthogonal, the clutter-plus-noise components are statistically independent and each component follows the log-normal distribution with parameters $(-0.45,0.67)$, Change the parameters of the Weibull reflection coefficients to $(1.04,10)$ and the result is shown by the solid curve with points marked with squares in Fig. 7.

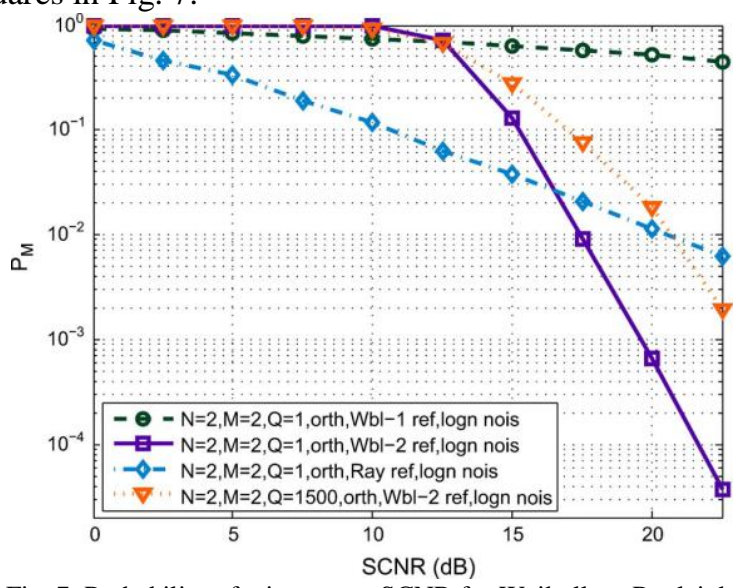

Fig. 7. Probability of miss versus SCNR for Weibull, or Rayleigh reflection coefficients and log-normal clutter-plus-noise

\section{Conclusion}

The diversity gains were analyzed for a signal-present versus signal-absent scalar hypothesis test statistic, a vector signal-present versus signal-absent hypothesis testing problem, and for a MIMO radar system. For each case, the Neyman-Pearson criterion is considered to obtain the optimal test statistic. Suboptimal tests were also discussed. We showed that, generally, the diversity gain is dependent on the lowest order power in an expansion, about zero, of the cdf of the signal part of the resultant test statistic, but invariant to the cdf of the clutter-plus-noise term in the test statistic under some reasonable conditions requiring certain moments of the magnitude of the processed clutter-plus-noise be bounded.

Specifically, for MIMO radar target detection, this paper extended the recent work in [1] by incorporating nonorthogonal waveforms, non-Gaussian reflections, and nonGaussian clutter-plus-noise. The Neyman-Pearson criterion is considered to obtain the optimal test statistic. By implementing diversity gain for MIMO NeymanPearson signal detection. It can be seen that the maximum achievable diversity gain remains constant for clutter plus noise CDF.

The target is composed of a sufficient number of statistically independent Gaussian scatterers, a Gaussian optimum detector is employed, and the antennas are properly placed, the maximum diversity gain can be achieved. Further proper non orthogonal waveform can achieve same diversity gain as orthogonal waveform.

\section{REFERENCES}

[1] R. S. Blum, "Limiting case of a lack of rich scattering environment for MIMO radar diversity," IEEE Signal Process. Lett., vol. 16, pp. 901-904, Oct. 2009.

[2] T. Aittomaki and V. Koivunen, "Performance of MIMO radar with angle diversity under Swerling scattering model," IEEE J. Sel. Topics Signal Process. (Special Issue on MIMO Radar), vol. 4, no. 1, pp.101-114, Feb. 2010. 
[3] H. Li and B. Himed, "Transmit subaperturing for MIMO radars," IEEE J. Sel. Topics Signal Process. (Special Issue on MIMO Radar), vol. 4, no. 1, pp. 55-65, Feb. 2010.

[4] Y. Yu, A. P. Petropulu, and H. V. Poor, "MIMO radar using compressive sampling," IEEE J. Sel. Topics Signal Process. (Special Issue on MIMO Radar), vol. 4, no. 1, pp. 146-163, Feb. 2010.

[5] A. Tajer, G. Jajamovich, and X. Wang, "Optimal joint target detection and parameter estimation by MIMO radar," IEEE J. Sel. Topics Signal Process. (Special Issue on MIMO Radar), vol. 4, no. 1, pp. 127-145, Feb. 2010.

[6] C.-Y. Chong, F. Pascal, J. P. Ovarlez, and M. Lesturgie, "MIMO radar detection in non-Gaussian and heterogeneous clutter," IEEE J. Sel.Topics Signal Process. (Special Issue on MIMO Radar), vol. 4, no. 1, pp. 115-126, Feb. 2010.

[7] Q. He, R. S. Blum, H. Godrich, and A. M. Haimovich, "Target velocity estimation and antenna placement for MIMO radar with widely separated antennas," IEEE J. Sel. Topics Signal Process. (Special Issue on MIMO Radar), vol. 4, no. 1, pp. 79-100, Feb. 2010.

8] Q. He, R. S. Blum, and A. M. Haimovich, "Noncoherent MIMO radar for location and velocity estimation: More antennas means better performance," IEEE Trans. Signal Process., vol. 58, no. 7, pp. 3661-3680, Jul. 2010. HE AND BLUM: DIVERSITY GAIN FOR MIMO NEYMAN-PEARSON SIGNAL DETECTION 881

[9] A. De Maio and M. Lops, "Design principles of MIMO radar detectors," IEEE Trans. Aerosp. Electron. Syst., vol. 43, pp. 886898, Jul. 2007.

[10] E. Fishler, A. M. Haimovich, R. S. Blum, L. Cimini, D. Chizhik, and R. Valenzuela, "Spatial diversity in radars-Models and detection performance," IEEE Trans. Signal Process., vol. 54, no. 3, pp. 823-838, Mar. 2006.

[11] A. M. Haimovich, R. S. Blum, and L. Cimini, "MIMO radar with widely separated antennas," IEEE Signal Process. Mag., vol. 25, no. 1, pp. 116-129, Jan. 2008.

[12] Q. He and R. S. Blum, "Cramer-Rao bound for MIMO radar target localization with phase errors," IEEE Signal Process. Lett., vol. 17, pp. 83-86, Jan. 2010.

[13] Q. He, N. Lehmann, R. S. Blum, and A. M. Haimovich, "MIMO radar moving target detection in homogeneous clutter," IEEE Trans. Aerosp. Electron. Syst., vol. 46, no. 3, pp. 1290-1301, Jul. 2010.

[14] R. Kaas, M. Goovaerts, J. Dhaene, and M. Denuit, Modern Actuarial Risk Theory: Using R, 2nd ed. New York: Springer, 2008.

15] G. A. Gibson, An Elementary Treatise on the Calculus: With Illustrations From Geometry, Mechanic and Physics. London, U.K.: MacMillan, 1922

[16] S. A. Kassam, Signal Detection in Non-Gaussian Noise. New York: Springer-Verlag, 1987.

17] E. Fishler, A. M. Haimovich, R. S. Blum, L. Cimini, D. Chizhik, and R. Valenzuela, "Spatial diversity in radars-Models and detection performance," IEEE Trans. Signal Process., vol. 54, no. 3, pp. 823-838, Mar. 2006

[18] Q. He, R. S. Blum, and A. M. Haimovich, "Noncoherent MIMO radar for location and velocity estimation: More antennas means better performance," IEEE Trans. Signal Process., vol. 58, no. 7, pp. 3661-3680, Jul. 2010.

[19] A. De Maio and M. Lops, "Design principles of MIMO radar detectors," IEEE Trans. Aerosp. Electron. Syst., vol. 43, pp. 886898, Jul. 2007.

[20] C. Chen and P. P. Vaidyanathan, "MIMO radar space-time adaptive processing using prolate spheroidal wave functions," IEEE Trans. Signal Process., vol. 56, no. 2, pp. 623-625, Feb. 2008.

[21] J. Li and P. Stoica, "MIMO radar with colocated antennas," IEEE Signal Process. Mag., vol. 24, no. 5, pp. 106-114, Sep. 2007.

[22] E. Fishler, A. M. Haimovich, R. S. Blum, L. Cimini, D. Chizhik, and R. Valenzuela, "Spatial diversity in radars-Models and detection performance," IEEE Trans. Signal Process., vol. 54, no. 3, pp. 823-838, Mar. 2006.

[23] H. L. Van Trees, Detection, Estimation, and Modulation Theory I. New York: Wiley, 2001.

[24] D. G. Luenberger and Y. Ye, Linear and Nonlinear Programming, 3rd ed. New York: Springer Science+Business Media, LLC, 2008

[25] S. A. Kassam, Signal Detection in Non-Gaussian Noise. New York: Springer-Verlag, 1987

[26] R. Kaas, M. Goovaerts, J. Dhaene, and M. Denuit

[27] D. G. Luenberger and Y. Ye, Linear and Nonlinear Programming, $3^{\text {rd }}$ ed. New York: Springer Science+Business Media, LLC, 2008.
[28] H. L. Van Trees, Detection, Estimation, and Modulation Theory I. New York: Wiley, 2001

[29] C. Chen and P. P. Vaidyanathan, "MIMO radar space-time adaptive processing using prolate spheroidal wave functions," IEEE Trans. Signal Process., vol. 56, no. 2, pp. 623-625, Feb. 2008.

[30] G. San Antonio, D. R. Fuhrmann, and F. C. Robey, "MIMO radar ambiguity functions," IEEE J. Sel. Topics Signal Process., vol. 1, no. 1, pp. 167-177, Jun. 2007.

[31] J. Li and P. Stoica, "MIMO radar with colocated antennas," IEEE Signal Process. Mag., vol. 24, no. 5, pp. 106-114, Sep. 2007. 